HEP/123-qed

\title{
Dynamic Rearrangements and Packing Regimes in Randomly Deposited Two-Dimensional Granular Beds
}

\author{
I. Bratberg用 \\ Department of Telecommunications, \\ Norwegian University of Science and Technology, N-7491 Trondheim, Norway. \\ F. Radjai \\ LMGC, CNRS-Université Montpellier II, \\ Place Eugne Bataillon, 34095 Montpellier cedex, France.
}

\author{
A. Hansen \\ Department of Physics, Norwegian University of \\ Science and Technology, N-7491 Trondheim, Norway.
}

(Dated: October 26, 2018)

\begin{abstract}
We study the structural properties of two-dimensional granular packings prepared by random deposition from a source line. We consider a class of random ballistic deposition models based on single-particle relaxation rules controlled by a critical angle, and we show that these local rules can be formulated as rolling friction in the framework of dynamic methods for the simulation of granular materials. We find that a packing prepared by random deposition models is generically unstable, and undergoes dynamic rearrangements. As a result, the dynamic method leads systematically to a higher solid fraction than the geometrical model for the same critical angle. We characterize the structure of the packings generated by both methods in terms of solid fraction, contact connectivity and anisotropy. Our analysis provides evidence for four packing regimes as a function of solid fraction, the mechanisms of packing growth being different in each regime.

PACS numbers: 83.80.Fg, 74.80.Bj, 81.05.Rm
\end{abstract}

*Temporary address: Fysisk Institutt, Universitetet i Oslo, Postboks 1048 Blindern, N-0316 Oslo, Norway 


\section{INTRODUCTION}

Random ballistic deposition (RBD) is a well-known method for layer-by-layer construction of random packings of hard particles such as granular beds and colloidal aggregates[1], 2]. This method is based on a simple and intuitive procedure. The particles (mainly monodisperse spheres or disks) are allowed to fall sequentially along randomly positioned vertical lines over a horizontal substrate. Upon contact with the substrate or the first (already deposited) particle, the particle either sticks or is further moved to a more favorable position according to a relaxation (or restructuring) rule. The RBD method can be efficiently implemented in a computer code for generating very large two- and three-dimensional packings. Elaborate large-scale simulations based on this approach have been used to investigate the geometrical properties of random packings (packing regimes, distribution functions, growth, etc) $[3,13$.

It is obvious that the random deposition of particles can also be simulated by means of dynamic methods, such as molecular dynamics [4, 5] and contact dynamics [6, 7, 8], in the spirit of a real experiment where the grains are poured into a box. Such simulations require, however, substantially more computation time 9]. This difficulty has been inhibiting enough to discourage for a long time systematic investigation of deposited beds following dynamic methods. But, the situation is far better today due to the fast increase of available computer power and memory during the last decade. There is now a considerable scope for dynamic simulations that can be exploited in order to study a number of highly interesting issues in the field of random packings.

The objective of the present paper is to apply a contact dynamics algorithm to investigate randomly-deposited granular beds in a two-dimensional geometry. The geometrical texture (coordination number, solid fraction, ... ) of a granular bed depends on several physical parameters (particle properties, contact interactions, inertia of deposited particles) which can be tuned in a dynamic simulation in order to characterize the impact of each parameter on the texture. We propose here an original approach which allows to bring out some interesting features of granular beds in comparison to RBD models. We consider a generalized RBD model in which the relaxation of the falling particle upon contact with the substrate is controlled by the direction of the contact normal $\theta$ (a single angle in 2D) 10. Depending on whether $\theta$ is below or above a critical angle $\theta_{c}$, the particle either simply sticks or is 
allowed to rotate until it reaches a local minimum position or forms a new contact below $\theta_{c}$. Hereafter, we refer to this model as the CA (Critical Angle) model. The central feature of this model is that it allows to control the solid fraction $\rho$ by varying the critical angle 10 . The approach we propose consists in performing dynamic simulations of random ballistic deposition as closely as possible to the CA model.

This requires that we transcribe the the above geometrical relaxation rule into a contact law which is reduced to the geometrical rule for the random deposition process. We first show that this requirement is met if the particles interact through a rolling friction law (similar to the Coulomb sliding friction) in which a contact torque is mobilized to resist relative rotation of two particles. We implement this law within a contact dynamics (CD) algorithm. Then, we perform two series of simulations both with the CA model and the CD method. In the first series, we use the granular beds prepared according to the CA procedure as initial configuration for a CD simulation. We show that, although local stability (sticking due to rolling friction or, alternatively, particles supported by two underlying contacts) is fulfilled for each particle added to the bed, the latter still undergoes collective rearrangements leading to a higher solid fraction. This implies that a granular packing prepared by geometrical rules is globally unstable. We study the extent of dynamic rearrangements and the structural properties of the CA and CD packings as a function of the critical angle.

In a second series of simulations, we characterize the packings in terms of the average coordination number, structural anisotropy and contact connectivity as a function of the solid fraction. We show that the trends are globally similar for the CD method and the CA model (the same packing regimes are observed). We distinguish several packing regimes where different mechanisms (screening, chaining, branching, piling, jamming and ordering) are active and control the packing fraction.

\section{NUMERICAL APPROACH}

\section{A. Critical-angle model}

Figure 1 shows the geometry of a contact formed by a falling particle $\mathrm{i}$ with a particle $\mathrm{j}$ of the substrate. The two particle centers define a line inclined at an angle $\theta_{i j}$ to the vertical. For disks, $\theta_{i j}$ is also the direction of the contact normal $\boldsymbol{n}_{i j}$, unit vector directed from the 
center of particle $\mathrm{j}$ to the center of particle i. For brevity, we will refer to $\theta_{i j}$ as the "contact direction".

The CA model is defined as follows [10]. If $\left|\theta_{i j}\right|$ is below a critical angle $\theta_{c}$, defined in the range between 0 (vertical direction) and $\pi / 2$ (horizontal direction), particle i simply freezes by sticking to particle $\mathrm{j}$. On the other hand, if $\left|\theta_{i j}\right|$ exceeds $\theta_{c}$, particle $\mathrm{i}$ is allowed to rotate around particle $\mathrm{j}$ until a second contact is formed with another particle $\mathrm{k}$ of the substrate. Then, there are three possible alternatives: 1) If $\left|\theta_{i k}\right|<\theta_{c}$, particle i freezes as in the first case; 2) If the new position of particle $\mathrm{i}$ is a local minimum position, again particle i freezes; 3) If neither of the two latter conditions is fulfilled, particle $\mathrm{i}$ is again allowed to rotate around particle $\mathrm{k}$ until a new contact is formed with another particle of the bed and the three alternatives are examined again with this new contact. This procedure is iterated until particle $\mathrm{i}$ is stabilized either by sticking or by reaching a local minimum position.

Two limits are of particular interest. When $\theta_{c}=90^{\circ}$, all particles stick irreversibly to the substrate where ever they land. This limit corresponds to the random sequential adsoption model [14, 15, 16, 17]. When $\theta_{c}=0^{\circ}$, all particles relax and the model is reduced to the steepest descent model [1], 12]. In [14], the solid fraction was found to be $\rho_{\min }=$ $0.3568 \pm 0.0001$ in the no-restructuring limit. The solid fraction for the steepest descent model is expected to come close to $\rho_{\max }=0.906$ corresponding to a triangular packing. However, to achieve a structure with long-range ordering, the initial conditions are very important. In practice, the bottom line must initially be covered by an array of contiguous disks. Otherwise, simulations using the steepest descent algorithm have shown that the solid fraction will not exceed $\rho=0.82$ which is the characteristic density of $2 \mathrm{D}$ monodisperse random close packing ( $\mathrm{RCP}$ ) where long-range order is broken by defects in the packing [14, 18].

In the CA model, the solid fraction $\rho$ of the granular bed is a function of the critical angle $\theta_{c}$ as shown in Fig. 2. In this figure, the solid fraction for each of the angles $\theta_{c}=0,1,2, \cdots, 90$ is an average over 30 independent runs. The bottom line was covered by a layer of 32 contiguous particles and 1000 particles were deposited in each run. In order to avoid wall effects, periodic boundary conditions were implemented in the horizontal direction. Let us note that CA simulations are possible at much larger scales. Nevertheless, we restrict here the size and the number of CA simulations to those reasonably accessible to dynamic simulations since the results will be compared between these two methods below. Figure 2 
shows that the solid fraction decreases monotonously from $\rho_{\max }$ to $\rho_{\min }$ as $\theta_{c}$ is increased from 0 to $90^{\circ}$. One can distinguish several regimes on this curve which will be discussed below in connection with dynamic simulations.

The CA model is a geometrical model that meets the no-overlap condition between hard particles but involves a number of physical approximations about the stability of the packing and its growth. By nature, this model neglects inertia effects. The substrate is frozen and the relaxation step involves only the deposited particle. Moreover, the two stability criteria (local minimum position and sticking condition) for the deposited particle have a local nature. In other words, the model assumes that the whole packing remains in static equilibrium as long as all particles are sequentially stabilized by either of these conditions.

In order to examine the validity of these assumptions, the approach followed in this paper is to perform dynamic simulations as closely as possible to the CA model and to compare the resulting packings. This implies that the particles should be released sequentially and they should hit the granular bed with negligibly small inertia. Moreover, upon contact with the substrate, the falling particle should dynamically stick or roll down, depending on the

value of the contact angle with respect to the critical angle, until one of the two stability conditions is fulfilled. There is no difficulty in tuning the inertia in a dynamic simulation. But, we need to define a dynamic version of the relaxation rule.

\section{B. Rolling friction}

The dynamic content of stability due to a local minimum position is clear. The weight of a particle can obviously be balanced by the reaction forces exerted by two underlying particles; see Fig. 3(a). But the sticking condition requires both a contact force $\boldsymbol{F}_{i j}$ and a "contact torque" $M_{i j}$ so as to counterbalance respectively the weight $m_{i} g$ of the deposited particle and its moment with respect to the contact point. Figure 3(b) illustrates this condition. The balance equations are

$$
\begin{aligned}
\boldsymbol{F}_{i j}+m_{i} \boldsymbol{g} & =0, \\
m_{i} g r_{i} \sin \theta+M_{i j} & =0,
\end{aligned}
$$

where $r_{i}$ is the particle radius and $\boldsymbol{g}$ is the gravity.

Let $N_{i j}$ and $T_{i j}$ be the components of the reaction force $\boldsymbol{F}_{i j}$ along and perpendicular to 
the contact normal $\boldsymbol{n}_{i j}$. From equations 2 one gets

$$
\begin{aligned}
N_{i j} & =m_{i} g \cos \theta, \\
T_{i j} & =m_{i} g \sin \theta, \\
M_{i j} & =r_{i} N_{i j} \tan \theta .
\end{aligned}
$$

The normal force $N_{i j}$ is positive (as it should) as long as $-\pi / 2<\theta<\pi / 2$ (the angles are measured from the vertical). On the other hand, the relative sliding is inhibited if $\left|T_{i j}\right| / N_{i j}<\mu_{s}$, where $\mu_{s}$ is the coefficient of (sliding) friction (or equivalently, $\theta<\theta_{s}$, where $\theta_{s}=\tan ^{-1} \mu_{s}$ is the angle of friction).

Now, if we require that particle i rolls only if $\theta \geq \theta_{r}$, then from equations we arrive at the following no-rolling condition:

$$
\frac{\left|M_{i j}\right|}{r_{i} N_{i j}}<\tan \theta_{r}=\mu_{r},
$$

where $\mu_{r}$ is a coefficient of rolling friction. Let us further assume that $M_{i j}$ remains equal to its threshold value $\pm \mu_{r} r_{i} N_{i j}$ when rolling occurs. This condition is similar to the sliding condition $T_{i j}= \pm \mu_{s} N_{i j}$.

The rolling friction law, as defined here, and the more familiar Coulomb (sliding) friction law are shown in Fig. 4 in the form of graphs [7, 20]. The rolling friction law relates the relative rotation velocity $\omega_{i j}=\omega_{i}-\omega_{j}$ of the two particles to the contact torque $M_{i j}$, whereas the sliding friction law relates the sliding velocity $v_{i j}^{s}$ to the tangential force $T_{i j}$. In fact, although for the sake of clarity we derived the condition 6 by considering the particular case of a deposited particle touching a particle of the bed, the application of rolling friction to a contact between two arbitrary particles in a packing is rather straightforward when formulated in the form of the graphs shown in Fig. 4 . The torque transmitted through a contact to a particle in static equilibrium, for example, is the torque necessary to balance the sum of all force moments and other contact torques acting on the particle in the same way as the mobilized torque $M$ in equations 6 counterbalances the moment $r m g \sin \theta$ of the particle weight.

The prescription of rolling friction in a dynamic method follows the same steps as the sliding friction. The relation between the contact torque and the relative rotation velocity (Fig. $4(\mathrm{a})$ ) can not be represented as a motived function. Hence, in the framework of the molecular dynamics method, based on explicit integration of the equations of motion, this 
friction law has to be replaced by an approximate function [7, 9, 21]. This "regularization" of the friction law is not necessary within the contact dynamics method which was employed for the present investigation[6, 7, 8].

Using either of these dynamic methods, the sticking condition can be achieved if $\mu_{s}$ is set to infinity (no sliding for no contact direction) and the angle of rolling friction $\theta_{r}$ is interpreted as the critical angle $\theta_{c}$. This was implemented in our contact dynamics simulations. Alternatively, one may set $\mu_{r}$ to infinity (no rolling for no contact direction) using the angle of sliding friction $\theta_{s}$ as the critical angle. It is also possible to use a combination of these two conditions. These conditions are not equivalent, but we will not discuss the differences in this paper. In all cases, the condition of sticking upon collision requires also a zero coefficient of restitution.

To summarize, the following conditions allow to perform a dynamic simulation of random particle deposition in close analogy with the CA model:

1. $\theta_{r}=\theta_{c}$

2. No sliding $\left(\theta_{s}=90^{\circ}\right)$

3. Weak inertia

\section{Zero coefficient of restitution}

The important difference is that, while in the CA model all degrees of freedom in the substrate are kinematically frozen, in our dynamic simulations only contact sliding is frozen by setting $\theta_{s}=90^{\circ}$. All other degrees of freedom are active and the rolling friction governs all contacts: the contact between the deposited particle and the bed, as well as all contacts in the bed. Since the particles are not frozen in the granular bed, sequential particle deposition may thus lead to rearrangements in the granular bed.

\section{Simulation parameters}

The contact dynamics (CD) simulations involve a number of parameters which should be adjusted so as to minimize inertia effects without loosing numerical efficiency. The largest inertia are produced by the largest head-on velocity $v_{\max }$ between colliding particles. Let $\Delta t$ be the time step. The contact force due to inertia produced by a collision is $m v_{\max } / \Delta t$. 
This force should be compared to the weight $m g$ of one particle. Hence, the dimensionless parameter characterizing the ratio of inertia to weights is

$$
\alpha=\frac{v_{\max }}{g \Delta t}
$$

The influence of $\alpha$ on the solid fraction and restructuring is an interesting subject in itself, but it will not be investigated in this paper. As emphasized previously, the focus here is put on those effects (equilibrium states, rearrangements) that arise from the geometrical configuration. Hence, we should use a low value of $\alpha$. However, lower values of $\alpha$ mean slower simulations. Hopefully, the framework of the CD method allows for large time steps $\Delta t$ up to the limitations related to the procedure of contact detection. On the other hand, the value of $v_{\max }$ can be imposed for the falling particles, but further relaxation inside the packing may produce large impact velocities. In particular, at low solid fractions, where large voids exist in the bed, the free fall of a particle over distances compared to the system height $H \simeq 60 r$ can give rise to impact forces far larger than the weight of a column of particles of the same height.

In order to avoid such strong uncontrolled inertia, we implemented a "velocity barrier" trick that limits the particle velocities to $v_{\max }=0,3 \mathrm{~ms}^{-1}$. With this choice, we can use a time step as large as $\Delta t=0.003 \mathrm{~s}$. Then, setting $g=100 \mathrm{~ms}^{-2}$, we get $\alpha=1$. This means that the largest impact force is just equal to the weight of a single particle. This choice is both reasonable and compatible with numerical efficiency.

\section{DYNAMIC REARRANGEMENTS}

\section{A. Stability of CA packings}

How stable are the granular beds prepared by means of the CA model? We have seen that the CD method, equipped with rolling friction together with suitable values of the parameters reducing inertia effects, meets the single-particle stability criteria of the CA model in the course of deposition, namely 1) the sticking condition as a function of the rolling friction angle $\theta_{r}$ (identified with the critical angle $\theta_{c}$ ), and 2) the local minimum position where the weight of a particle is balanced by the reaction forces at the two underlying contacts. Now, if we start a CD simulation using a packing constructed according to the CA model as the initial configuration, then one of the two following alternatives may occur. 
If the single-particle stability criteria used in the course of deposition provide a sufficient condition for the global stability of the packing when the deposition is over, then the packing will remain in static equilibrium and the calculated forces will exactly balance all particles. Otherwise, the packing will be unstable and the $\mathrm{CD}$ simulations allow to calculate the particle rearrangements until a relaxed stable configuration is obtained.

Our simulation data confirm rather the second alternative for nearly all values of the critical angle. One example is shown in figures 5 (a) and 5 (b) for a packing of 500 particles with $\theta_{c}=40^{\circ}$. The rearrangements occur in the whole bed, but they are much more hindered in the bulk than in the vicinity of the free surface. For this reason, the displacements appear mostly in the uppermost layers. The relaxed configuration has a larger packing friction. The solid fraction is still larger when the CD simulation is performed by random deposition of the same sequence of particles (as in the CA simulation) for the same value of $\theta_{c}$ (and the same boundary conditions), instead of using the CA configuration as the initial condition.

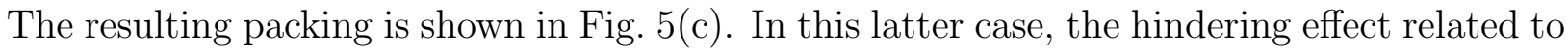
the bulk density, which was active in the case (b), disappears since the CD rearrangements occur naturally in the course of deposition for each deposited particle. This means that the degree of instability of the CA packing shown in Fig. 5(a) is more keenly reflected in the increase $\Delta \rho$ of the solid fraction from (a) to (c) than from (a) to (b).

Figure 6 displays the solid fraction $\rho$ as a function of $\theta_{c}$ for packings prepared by CD sequential random deposition. The solid fraction for each value of $\theta_{c}$ is an average over 10 independent $\mathrm{CD}$ runs. The CD simulations were performed for 40 different angels. The curve of $\rho$ as a function of $\theta_{c}$ corresponds thus to $410 \mathrm{CD}$ simulations of 1000 particles. Figure 6 shows that, as expected, the solid fraction is every where larger for the CD method than for the CA model, except at $\theta_{c}=0$ where a dynamic method requires an exceptionally high precision to reach a perfect triangular packing. Indeed, in this limit, tiny fluctuations in particle positions around a particle due to numerical overlaps are exponentially amplified in space as a result of long-range order 19]. Disregarding this pathological limit, the difference $\Delta \rho$ is negligibly small for $\theta_{c}<20^{\circ}$ and $\theta_{c}>80^{\circ}$. The largest variation $\Delta \rho$ of the solid fraction, representing the largest dynamic rearrangements in the packing, occurs at $\theta_{c} \simeq 50^{\circ}$, where $\rho$ increases from 0.45 for the CA packing to 0.6 for the CD packing. 


\section{B. Influence on the packing structure}

The coordination number $z$ (average number of contact particles around a particle), shown in Fig. 7 as a function of $\theta_{c}$, follows the same trends as the solid fraction. It is systematically larger in a CD packing than in the corresponding CA packing (for the same value of $\theta_{c}$ ) except in the $\theta_{c}=0$ limit. A coordination number close to 2 reflects the predominance of particle "chains" in a highly porous packing. The coordination number increases from 2 to 3 due to "branching", and from 3 to 4 due to a growing interplay of chains. The increase of $z$ beyond 4 requires long-range ordering 22]. This transition occurs only in the CA packing where the numerical precision is less stringent than in dynamic simulations.

Due to dynamic rearrangements in $\mathrm{CD}$ random deposition, the CA and CD packings show also very different aspects as to the directional order of the contact network. The nonuniform distribution of contact directions can be characterized by means of the fabric tensor $\boldsymbol{\phi}$ defined from contact normals $\boldsymbol{n}^{k}=\left(\sin \theta^{k}, \cos \theta^{k}\right)$ by 23, 24]

$$
\phi_{\alpha \beta}=\frac{1}{N_{c}} \sum_{k=1}^{N_{c}} n_{\alpha}^{k} n_{\beta}^{k},
$$

where $N_{c}$ is the total number of contacts, and $n_{\alpha}^{k}$ (resp. $n_{\beta}^{k}$ ) is the $\alpha$ (resp. $\beta$ ) component of the contact normal k. When the probability distribution function $p(\theta)$ of contact directions is known, the fabric tensor can be calculated from the integral

$$
\phi_{\alpha \beta}=\int_{-\pi / 2}^{\pi / 2} n_{\alpha}^{k} n_{\beta}^{k} p(\theta) d \theta .
$$

By construction, we have $\phi_{1}+\phi_{2}=\operatorname{tr}(\phi)=1$, where $\phi_{1}$ and $\phi_{2}$ are the eigenvalues. The mean contact direction in the packing is given by the major principal direction $\theta_{f}$ of $\boldsymbol{\phi}$. The structural anisotropy of the packing is represented by $a=2\left(\phi_{1}-\phi_{2}\right)$. The factor 2 is introduced in order to identify this value of $a$ with that appearing naturally in a sinusoidal distribution $p(\theta)=(1 / \pi)\left\{1+a \cos 2\left(\theta-\theta_{f}\right)\right\}[25]$.

Figure 8 shows the anisotropy of our granular beds as a function of $\theta_{c}$. We see that the anisotropy of CD packings is systematically below that of CA packings. This is mainly because collective rearrangements tend to destroy columnar structures in a CD packing. In

both cases, the anisotropy comes very close to zero for $\theta_{c}=0$. This effect is mainly related to the presence of a great number of particles with 5 and 6 contacts. In fact, using Eq. 8, it 
can be shown that the anisotropy for the set of 6 contacts around a particle is zero, and for a set of 5 contacts around a particle can not exceed a threshold imposed by steric exclusions.

The largest anisotropy in the CD packings is reached for $\theta_{c}=90^{\circ}$, whereas the anisotropy of the CA packing passes through a maximum at $\theta_{c} \simeq 50^{\circ}$. The anisotropy can be estimated analytically at $\theta_{c}=90^{\circ}$ where the packing growth is governed by sticking. Since the particles are released at random horizontal positions, the probability that a particle sticks at a contact angle $\theta$ (with respect to the vertical) is $p(\theta)=\frac{1}{2} \cos \theta$. Note that the latter is a normalized probability density function over the range $[-\pi / 2, \pi / 2[$. Using Eq. 9 with this expression for $p(\theta)$, we find $a\left(\theta_{c}=90^{\circ}\right)=2 / 3$ which is consistent with both CD and CA results at $\theta_{c}=90^{\circ}$ shown in Fig. 8.

Since $p(\theta)$ is an even function of $\theta(p(\theta)=p(-\theta))$, the major principal direction of the fabric tensor is vertical $\left(\theta_{f}=0\right)$. However, this is only a consequence of symmetry and it does not imply that the distribution $p(\theta)$ is peaked on $\theta=0$. In fact, within each of the half-intervals $[-\pi / 2,0]$ and $[0, \pi / 2]$, the contacts have preferred directions. This can be seen in one example of $p(\theta)$ for $\theta_{c}=0$ shown in Fig. 9. We observe a local maximum at $\theta=0$, but there are local maxima also in each of the half-intervals. In order to extract the useful information about the direction of contacts, one can calculate the fabric tensor $\phi$ by restricting the definition to one of the above two half-intervals.

The major principal direction $\theta_{f}$ for the interval $[0, \pi / 2]$ as a function of $\theta_{c}$ is displayed in Fig. 10. At $\theta_{c}=0$ and $\theta_{c}=90^{\circ}$ both methods give the same direction, but every where else the contacts are more biased to the horizontal direction in CD simulations compared to CA simulations. This is an indication that the collective rearrangements reorganize contact directions. As for the anisotropy, the value of $\theta_{f}$ can be calculated analytically in the limit $\theta_{c}=90^{\circ}$ over the interval $[0, \pi / 2]$ from the fabric tensor. The distribution function normalized over this interval is given by $p(\theta)=\cos \theta$ and the integral in Eq. 9 is calculated over the same interval. We find $\theta_{f}\left(\theta_{c}=90\right) \simeq 32^{\circ}$, in agreement with the simulation result shown in Fig.10.

The existence of a local minimum in the evolution of $\theta_{f}$ for the CA model at $\theta_{c} \simeq 30^{\circ}$ or the changing of behavior in the CD curve at the same point can be understood as a consequence of competition between sticking and rolling. As $\theta_{c}$ is increased from zero, an increasing number of particles stick to the substrate at an angle in the interval $\left[0, \theta_{c}\right]$. These include both the ones sticking to the bed upon the first collision (whose number increases 
as $\left.\int_{0}^{\theta_{c}} \cos \theta d \theta=\sin \theta_{c}\right)$ and a number of the relaxed particles. On average, this subset of contacts tends to decrease $\theta_{f}$ as long as $\theta_{c}$ is not too large. This explains the decrease of $\theta_{f}$ from $45^{\circ}$ at $\theta_{c}=0$ to $\simeq 28^{\circ}$ at $\theta_{c} \simeq 30^{\circ}$. But, the inclination of the contacts to the vertical increases at the same time following the increase of $\theta_{c}$. This trend dominates clearly the evolution of $\theta_{f}$ beyond $\theta_{c}=30^{\circ}$.

\section{PACKING REGIMES}

The results presented in the last section show that, for a given value of the critical angle $\theta_{c}$, the solid fraction and the structure of the packing differ considerably from the CA model to the CD approach (excepted in the two limits of very loose and very dense packings). We attributed these differences to dynamic restructuring in CD packings as the particles are added to the substrate. However, in this section, we will show that the structure of a CA packing is quite similar to that of a CD packing if they are compared at the same solid fraction $\rho$ (and thus, for different critical angles). This means that the structural properties of CA packings are quite realistic (close to CD packings) when they are considered as a function of the solid fraction rather than the critical angle.

\section{A. Fabric}

Figure 11 shows the coordination number $z$ as a function of solid fraction $\rho$ for CA and CD packings. In both cases, $z$ increases with $\rho$. The two curves almost collapse for $\rho<0.6$. For $0.6<\rho<0.8$, the CD packings show only a slightly larger coordination number than the CA packings. For $0.8<\rho$, the CD packings show a slightly lower coordination number than the CA packings. The solid fraction $\rho=0.8$ corresponds to $z \simeq 4$ in both methods.

The anisotropy $a$ of the packings as a function of $\rho$ is displayed in Fig.12. The anisotropy decreases as a function of $\rho$ for both methods except in the loosest CA packings where it increases a bit with $\rho$ and passes through a peak before decreasing. The relatively low rate of decrease in the range $\rho<0.6$ suggests that sticking is the dominant mechanism of growth in this regime, whereas rolling (or relaxation) is far more efficient in the subsequent range.

Figure 13 shows the major principal direction $\theta_{f}$ of the fabric tensor restricted to the interval $[0, \pi / 2]$ (as defined in the last section) as a function of $\rho$. In CD packings, the 
direction $\theta_{f}$, representing the average direction of contact normals in the interval $[0, \pi / 2]$, increases quite slowly for $\rho<0.6$ and much faster beyond 0.6. In CA packings, the anisotropy decreases for $\rho<0.6$, passes through a minimum at $\rho \simeq 0.6$ and increases in the subsequent range. However, let us remark that, as for the anisotropy, the difference in the value of $\theta_{f}$ between the two methods as a function of $\rho$ is quite small as compared to the differences as a function of the critical angle (see Fig. 10).

\section{B. Connectivity}

The coordination number $z$ is an average over all particles in a packing. But, the number of contact neighbors $q$ varies in a packing from particle to particle. In a monodisperse pile, $q$ can vary from 1 to 6 . This "connectivity disorder" characterizes the disposition of the particles as "nodes" of the contact network. The connectivity of a packing is given by the fraction $P_{q}$ of particles having $q$ contact neighbors. $P_{1}$ corresponds to the "dead ends" of particle chains. The larger $P_{1}$, the stronger "screening" (the dead ends did not grow because they were screened by faster growing structures). $P_{2}$ and $P_{3}$ are related to chaining and branching, respectively. $P_{4}$ corresponds to "piling", i.e. a natural situation where a particle is supported by two underlying particles and supports two others. $P_{5}$ and $P_{6}$ define "jammed" and ordered configurations.

Figure 14 shows the connectivity numbers $P_{q}$ as a function of solid fraction for $q$ varying from 1 to 6. The trends are globally similar in CA and CD packings and the differences for the two methods are quite small. All connectivity numbers vary monotonously with solid fraction except $P_{3}$ which first increases to reach a maximum at $\rho \simeq 0.7$ and decreases rapidly afterwards. In the range $\rho<0.6, P_{3}$ and $P_{4}$ increase at the expense of $P_{1}$ and $P_{2}$ which decrease. $P_{5}$ and $P_{6}$ begin to increase significantly only at $\rho \simeq 0.7$ and $\rho \simeq 0.8$, respectively.

The connectivity diagram $P_{q}$ is shown for three different solid fractions in Fig.15. The largest connectivity number is 2 for $\rho<0.6,3$ for $0.6<\rho<0.7$, and 4 for $0.7<\rho$. Interestingly, the screening effect is more important in CD simulations (the CD curve for $P_{1}$ stands above the corresponding CA curve). Chaining $\left(P_{2}\right)$, branching $\left(P_{3}\right)$ and ordering $\left(P_{6}\right)$ are slightly less important in $\mathrm{CD}$ simulations, while piling $\left(P_{4}\right)$ and jamming $\left(P_{5}\right)$ are enhanced.

The above data show that the morphology of a CA packing is very close to that of a CD 
packing at the same solid fraction. Both methods suggest four packing regimes characterized by the properties of the packing structure as a function of solid fraction:

(a) $\rho<0.6$ : This regime corresponds to loose random packings characterized by chaining (i.e. $P_{2}$ is the largest connectivity number), branching $\left(P_{3}\right.$ increases as a function of $\rho$ and becomes dominant at $\rho=0.6)$ and screening ( $P_{1}$ is large).

(b) $0.6<\rho<0.7$ : This is the regime of moderate random packings characterized by the largest value of $P_{3}$ (branching) at the expense of chaining $\left(P_{2}\right)$ which decreases rapidly as a function of $\rho$.

(c) $0.7<\rho<0.8$ : This regime corresponds to dense close packings where piling is the main mechanism of growth and $P_{4}$ is larger than other connectivity numbers.

(d) $0.8<\rho$ : This is the well-known dense ordered packing regime 18 characterized by $z>4$.

The transition densities $(0.6,0.7$ and 0.8$)$ appearing in this classification are approximate values. A more refined evaluation of these specific densities requires a considerably more computation time and a deeper insight into the mechanisms at play during the packing growth.

\section{CONCLUSION}

We investigated the structure of a class of randomly deposited granular packings whose density is controlled by a geometrical parameter, referred to as the critical angle. We used both a random ballistic deposition model with simple relaxation rules (the CA model), and a contact dynamics algorithm (the CD method) that incorporates those relaxation rules through a rolling friction law. The CD approach naturally leads to stable packings following dynamic rearrangements while in the $\mathrm{CA}$ model the packing is kinematically frozen after each single-particle relaxation.

The following results were shown by means of extensive simulations:

1) The packings prepared according to the CA model are generically unstable. When fed into the CD algorithm as initial configuration, the CA packings undergo dynamic 
rearrangements. As a consequence, the solid fraction is larger in CD packings than in CA packings for the same critical angle (implemented as the angle of rolling friction in the framework of the contact dynamics method).

2) The structural properties (anisotropy, connectivity) are quite comparable in CA and CD packings for the same solid fraction, even though significant differences were observed in packing anisotropies.

3) Both methods reveal four packing regimes as a function of solid fraction, the prevailing mechanism of growth being different in each regime.

An important outcome of this work is to show that the dynamic rearrangements are quite weak in the very loose and very dense limits where the structural properties are nearly the same. This means that, the random sequential adsorption model (irreversible sticking without relaxation), leading to very loose packings, and the steepest descent model (no sticking), leading to very dense packings, can be used with confidence in these two limits.

The CD simulations reported in this work were meant to keep as close as possible to the CA model in order to perform comparable calculations with both methods. There is much more to be learned about the structure of particle packs generated by the CD method (or equivalently, molecular dynamics method). The influence of inertia and polydispersity on the observed packing regimes is currently under investigation. The shear resistance of deposited beds (e.g. in a biaxial compression) as a function of solid fraction is another important issue which we would like to address in near future.

\section{ACKNOWLEDGMENTS}

We thank Knut Jørgen Måløy , Anders Malthe Sørensen, Espen Gjettestuen and Lothar Brendel for fruitfull discussions. This work had it's financial support by the Research Council of Norway (NFR) through a Strategical University Program. We are also grateful to the CNRS and NFR for support through the Franco-Norwegian PICS program, Grant No. 753.

[1] R. Jullien, P. Meakin and A. Pavlovitch, in Disorder and Granular Media, edited by D. Bideau and A. Hansen (North-Holland, Amseterdam, 1993) p. 103. 
[2] D. Houi, Hydrodynamics of dispersed media Editors J.P.Hulin, A.M. Cazabat, E.Guyon, F.Carmona, Elsevier Science Publishers B.V (1990)

[3] R. Jullien, P. Jund and D. Caprion, Phys. Rev. E 54, 6035 (1996).

[4] M.P. Allen and D.J. Tildesley, Computer simulation of liquids, (Oxford Science Publications, New York, 1997).

[5] S. Luding in Physics of Dry Granular Media (NATO ASI Series) edited by H.J. Herrmann, J.-P. Hovi and S. Luding (Kluwer Academic Publishers, Dordrecht, 1998) p. 285.

[6] J.J. Moreau, Eur. J. Mech. A/Solids 13 (nº 4-suppl.), 93 (1994).

[7] M. Jean, in Mechanics of Geometrical Interfaces, edited by A.P.S. Selvaduri and M.J. Boulon (Elsevier, Amsterdam, 1995) p. 463.

[8] F. Radjai, Computer Physics Communications 121-122, 294 (1999).

[9] J. Schäfer, S. Dippel and D.E. Wolf, J. Phys. I France 6, 5 (1996).

[10] P.K. Watson, H. Mizes A. Castellanos, A. Mizes, in Powders and Grains 97 edited by R. P. Behringer et J. T. Jenkins (Balkema, Rotterdam, 1997) p.109.

[11] E.M. Tory, N.A. Cochrane, S.R. Waddell, Nature (London) 2201023 (1968).

[12] W.M. Visscher and M. Bolsterli, Nature (London) 239504 (1972).

[13] R. Jullien and P. Meakin, Europhys Lett. 6629 (1988).

[14] P.Meakin and R.Jullien, J. Physique 46, 1543 (1985).

[15] M.J. Vold, J. Colloid Sci. 14,168 (1959); J. Phys. Chem. 63, 1608 (1959); bf 64, 1616 (1960).

[16] B.D. Lubachevsky, V. Privman and S.C. Roy, Phys. Rev. E 47, 48 (1993).

[17] C. Tang and S. Liang, Phys. Rev. Lett. 71, 2769 (1993).

[18] T.I. Quickenden and G.K. Tan, J. Colloid Interface Sci. 48, 382 (1974).

[19] J.G. Berryman, Phys. Rev. A 27, 1053 (1983).

[20] F. Radjai and S. Roux, Phys. Rev. E 51, 6177 (1995).

[21] F. Radjai, J. Schäfer, S. Dippel and D. Wolf, J. Phys. I 7, 1053 (1997).

[22] A. Gervois and D. Bideau, in Disorder and Granular Media, edited by D. Bideau and A. Hansen (North-Holland, Amseterdam, 1993) p. 1.

[23] M. Satake, in Continuum-mechanical and statistical approaches in the mechanics of granular materials edited by S. C. Cowin and M. Satake (Gakujutsu Bunken Fukyu-kai, Tokyo, 1978) p. $47-62$.

[24] F. Radjai, D. Wolf, M. Jean, et J. J. Moreau, Phys. Rev. Lett. 90, 61 (1998). 
[25] L. Rothenburg and R. J. Bathurst, Géotechnique 39, № 4, 601-614 (1989). 


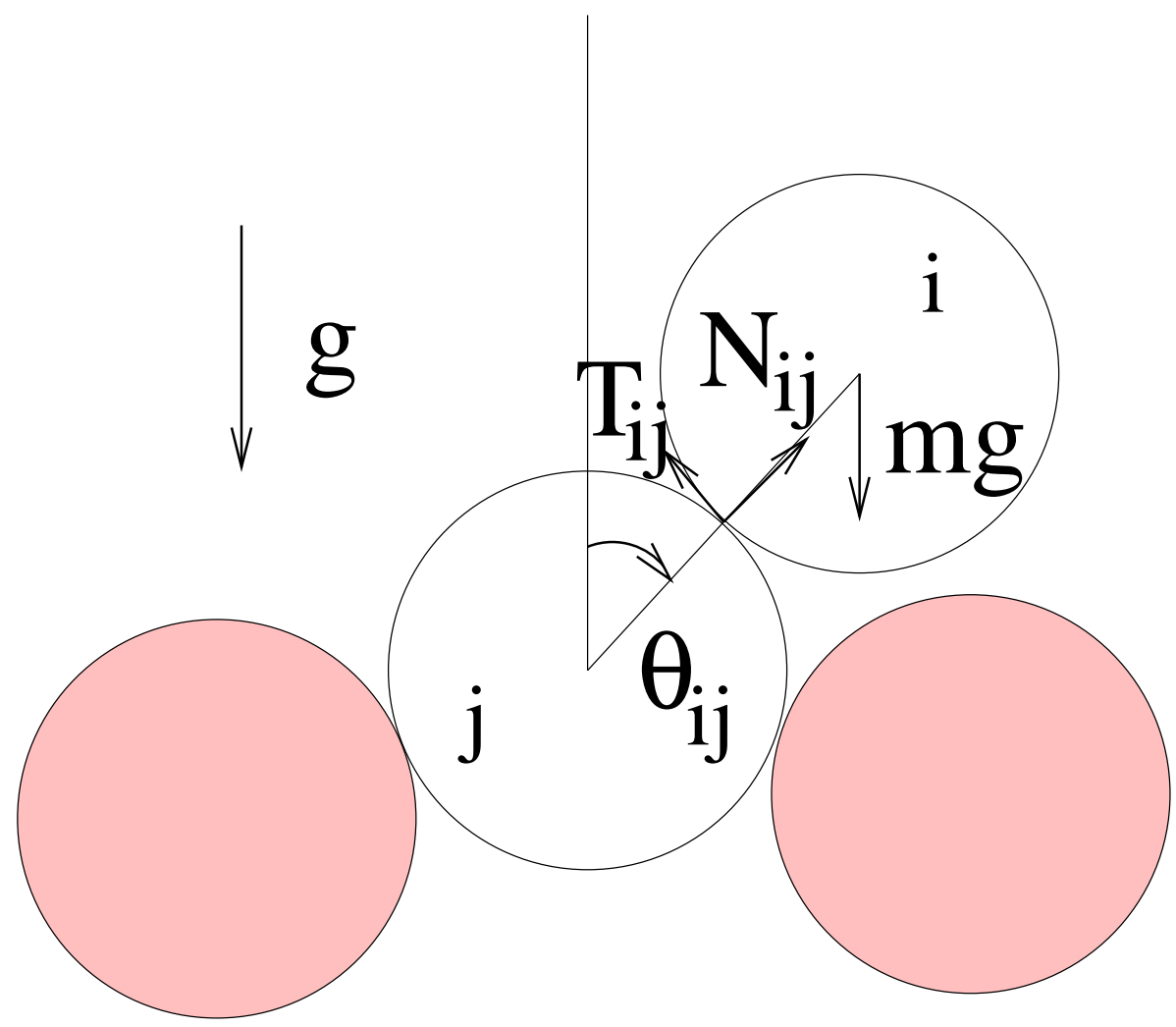

FIG. 1: Geometry of a contact formed by a falling particle i with a particle $\mathrm{j}$ from the substrate. The contact angle $\theta_{i j}$ is measured from the vertical. 


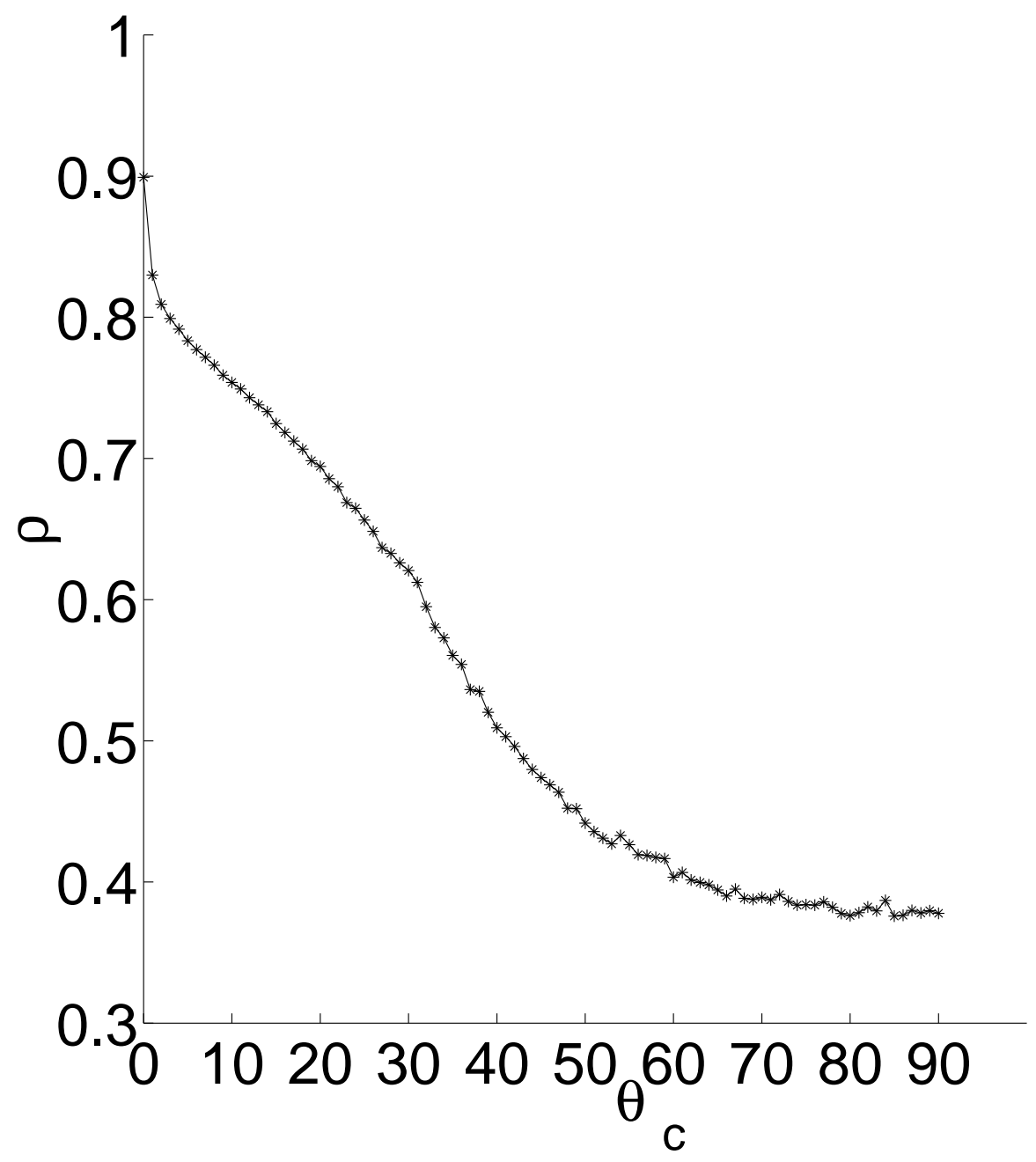

FIG. 2: Solid fraction $\rho$ as a function of critical angle $\theta_{c}$ (in degrees) for the CA model. 


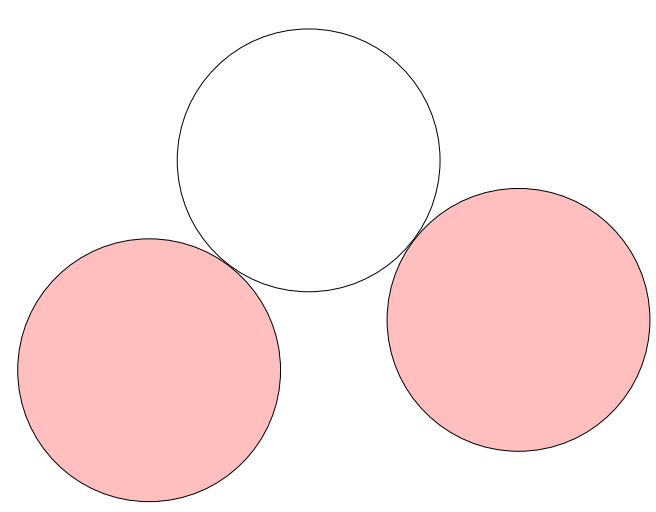

(a)

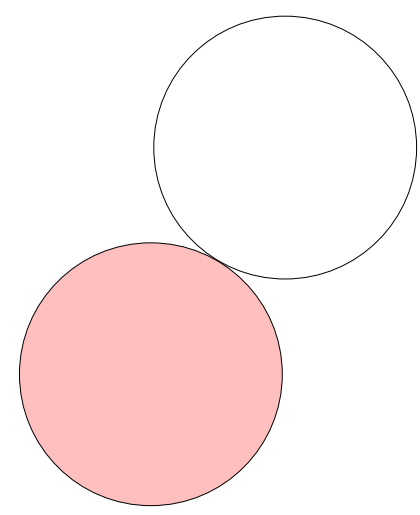

(b)

FIG. 3: The two local stability conditions in the CA model: (a) local minimum position; (b) sticking. 


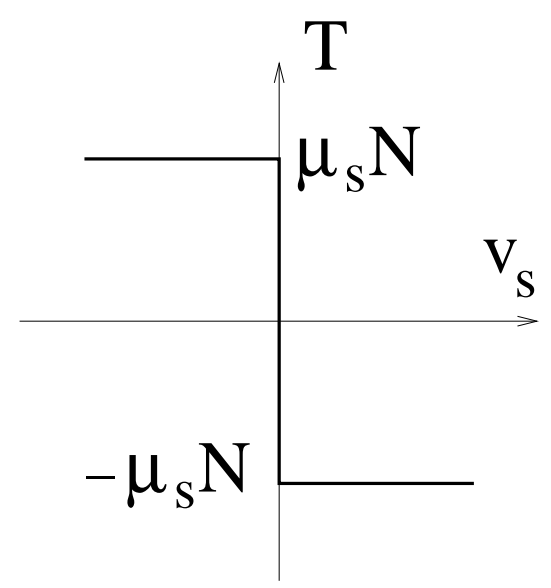

(a)

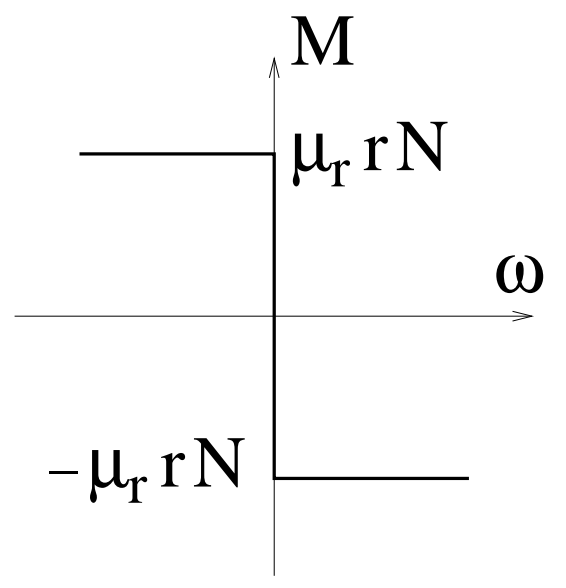

(b)

FIG. 4: The graphs representing (a) sliding friction law and (b) rolling friction law; see text. 
(a)

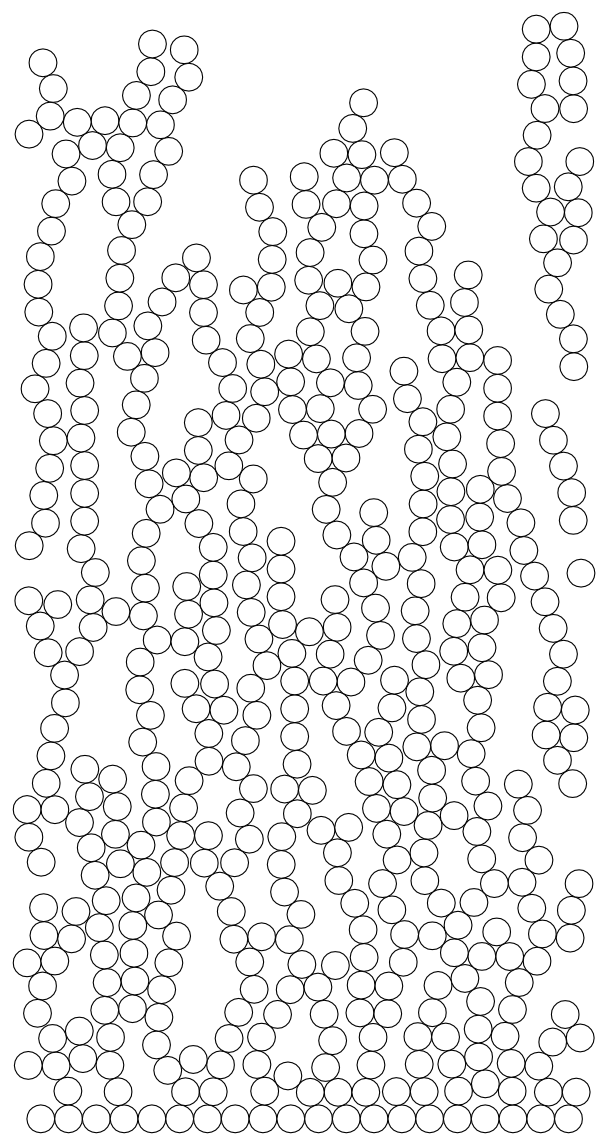


(b)

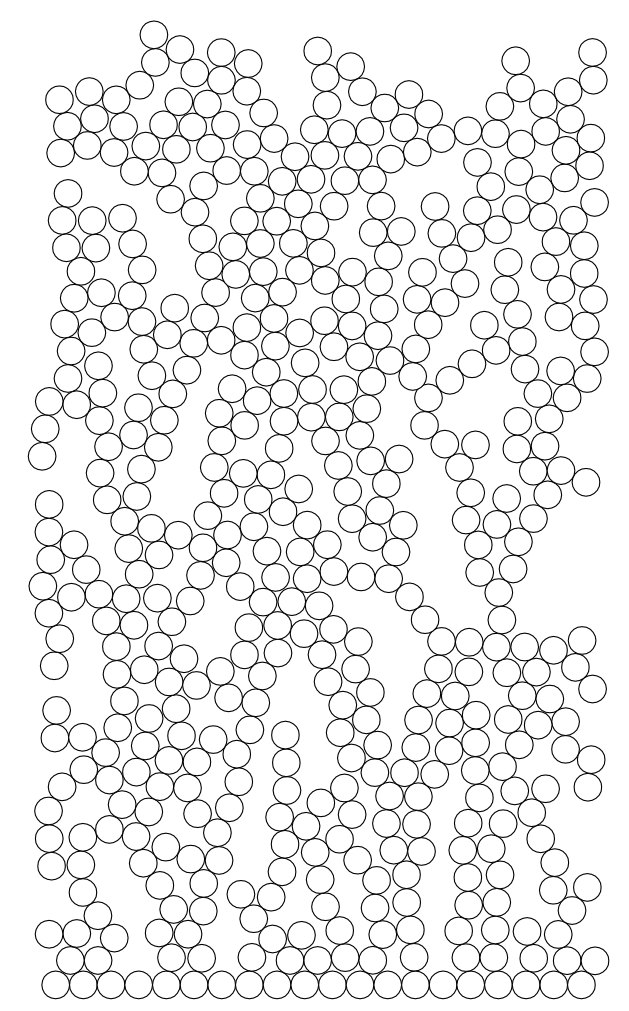




\section{( c )}

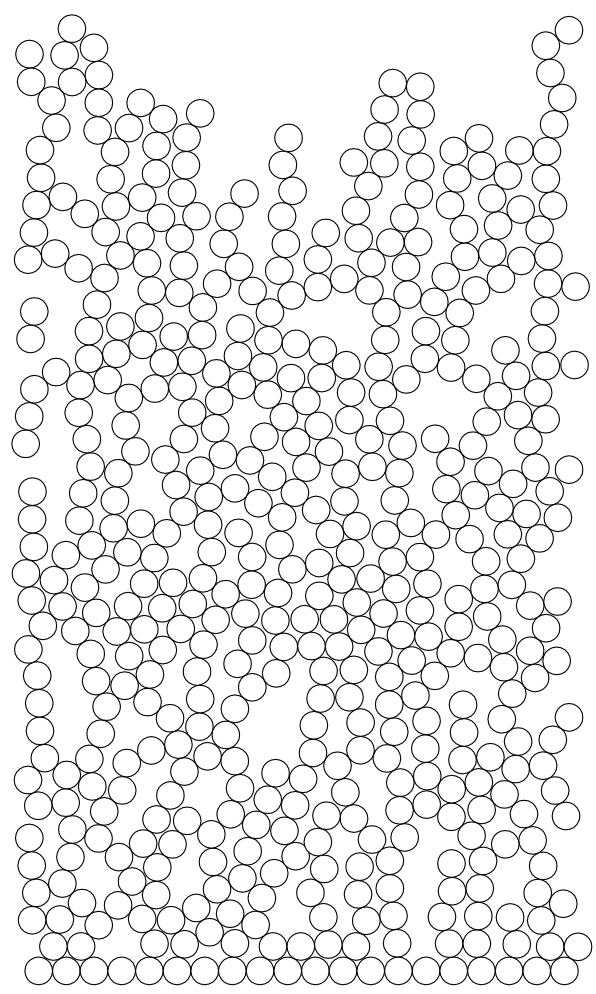

FIG. 5: (a) An example of a CA packing; (b) The static packing obtained by the CD method starting with the CA packing in (a) as initial condition; (c) CD packing obtained by using the same sequence of falling particles as in (a). 


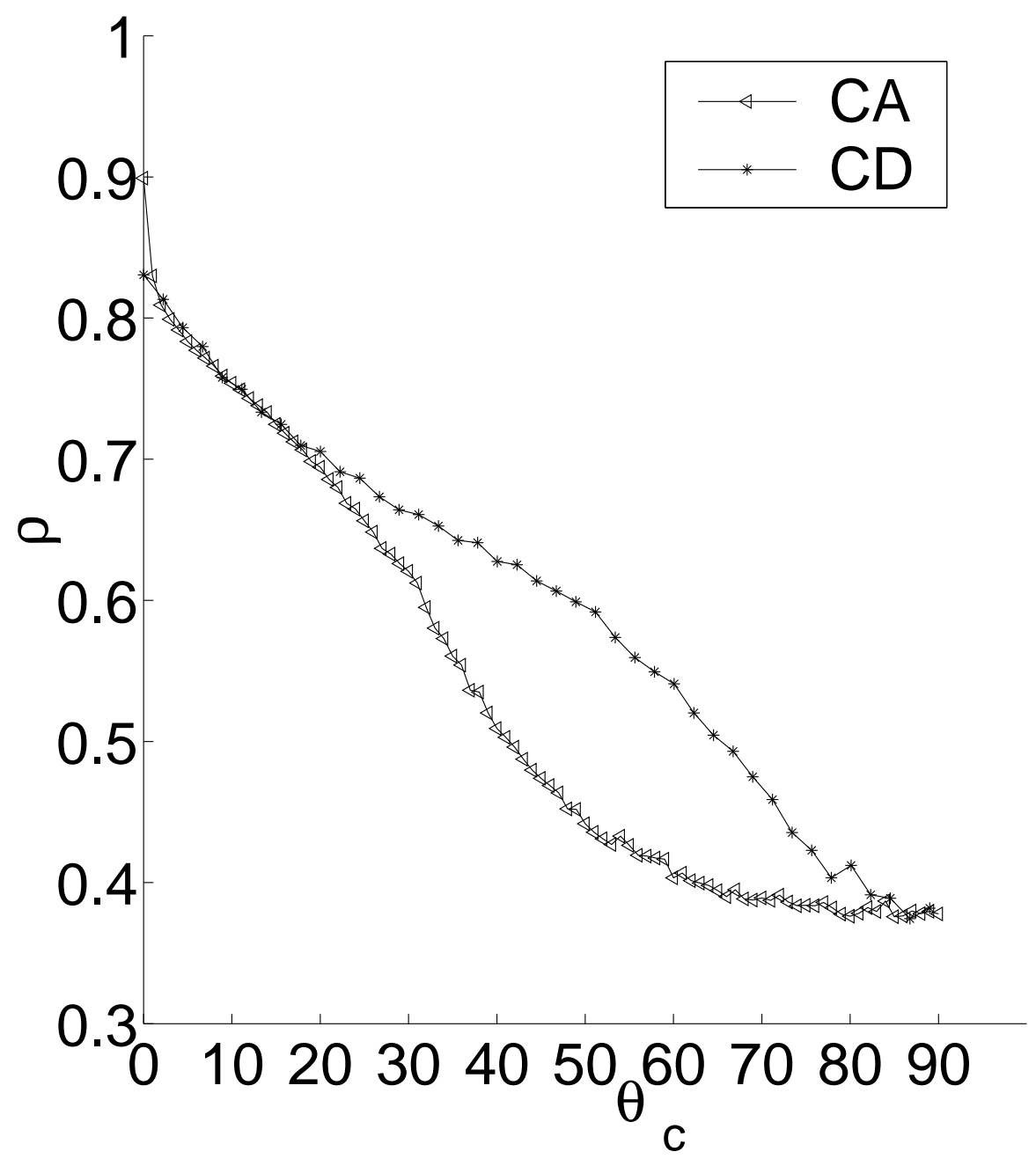

FIG. 6: Solid fraction as a function of critical angle (in degrees) for the CD and CA models. 


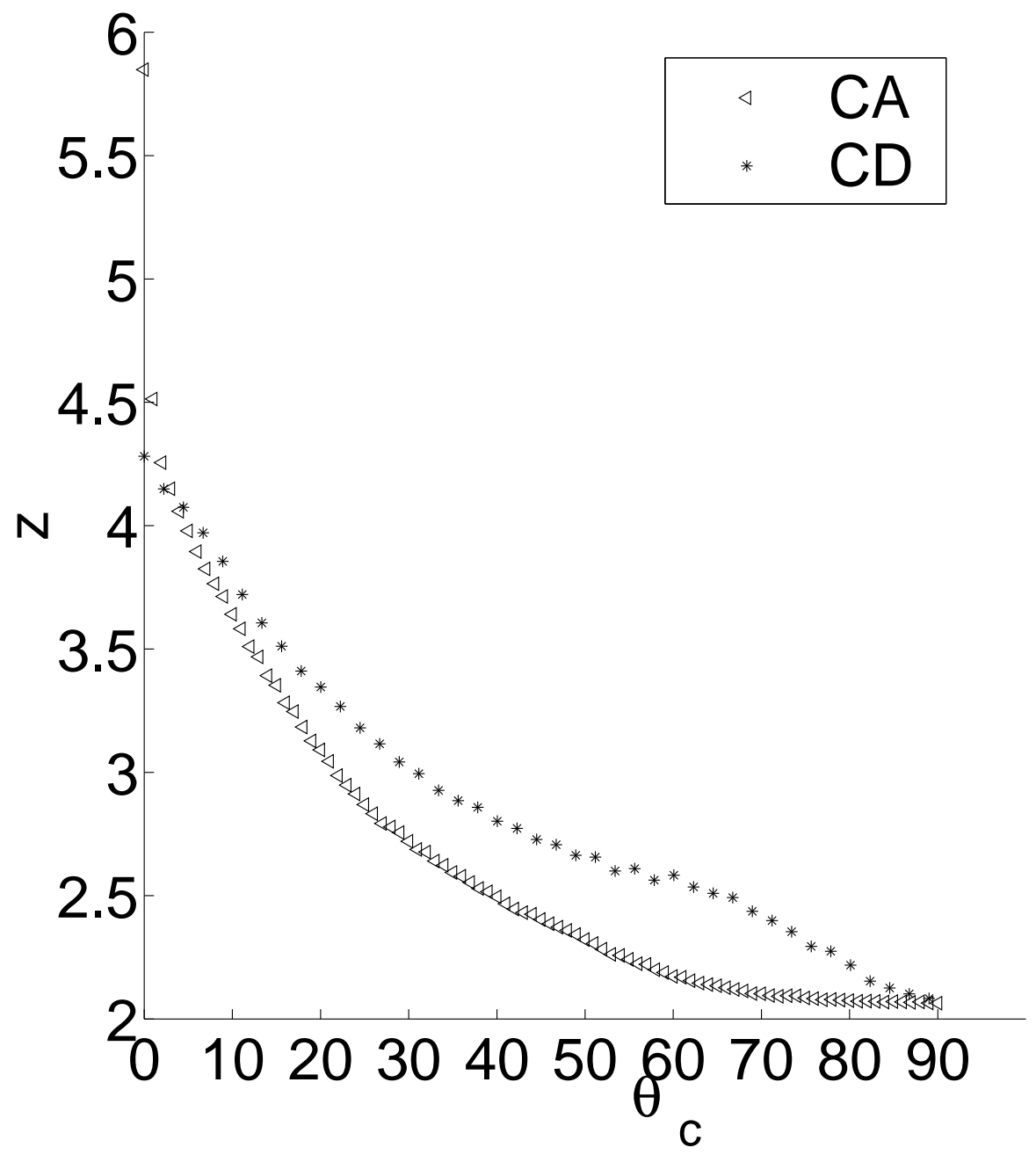

FIG. 7: Coordination numbers $z$ as a function of critical angle (in degrees). 


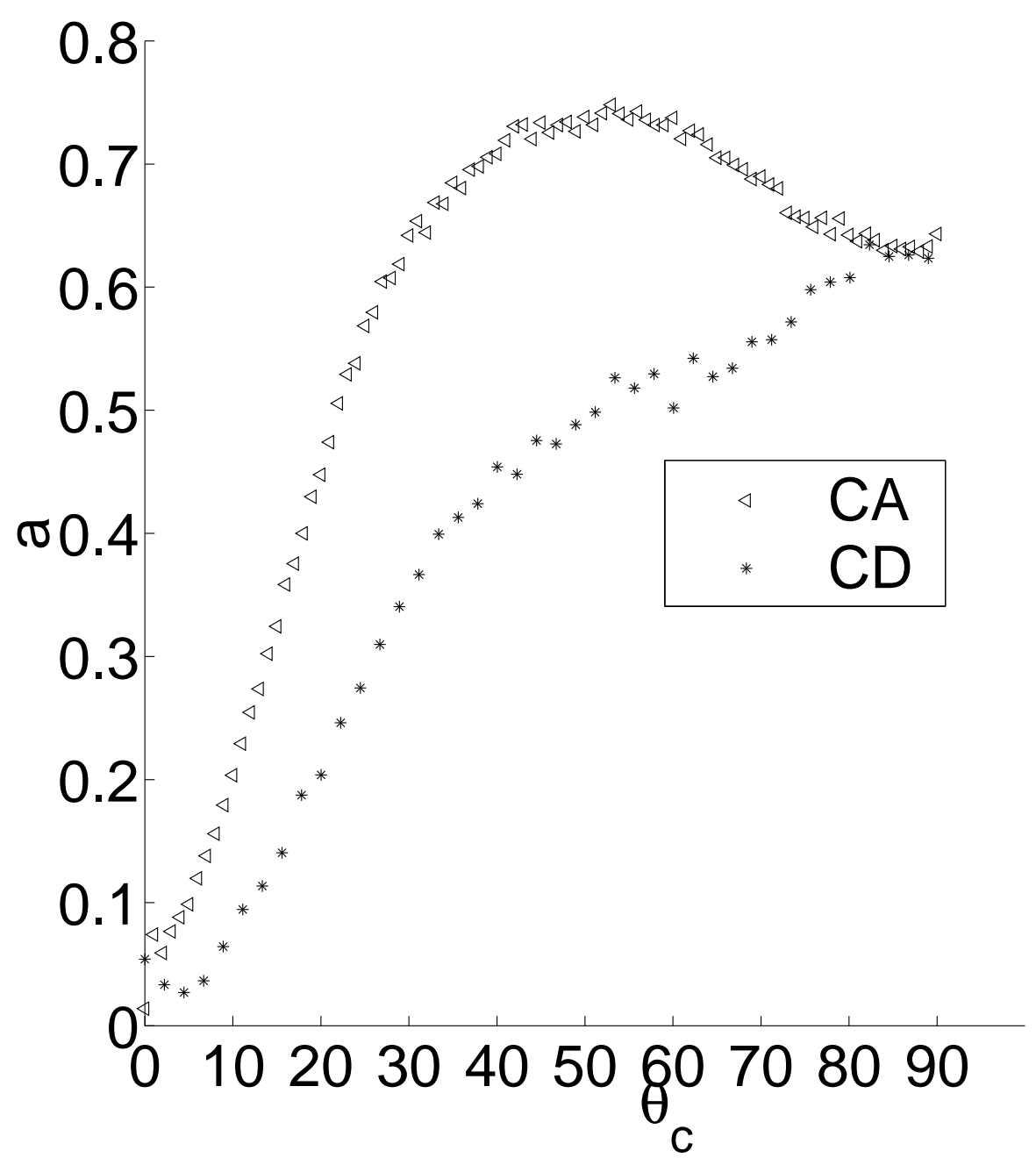

FIG. 8: Anisotropies $a$ as a function of critical angle (in degrees). 


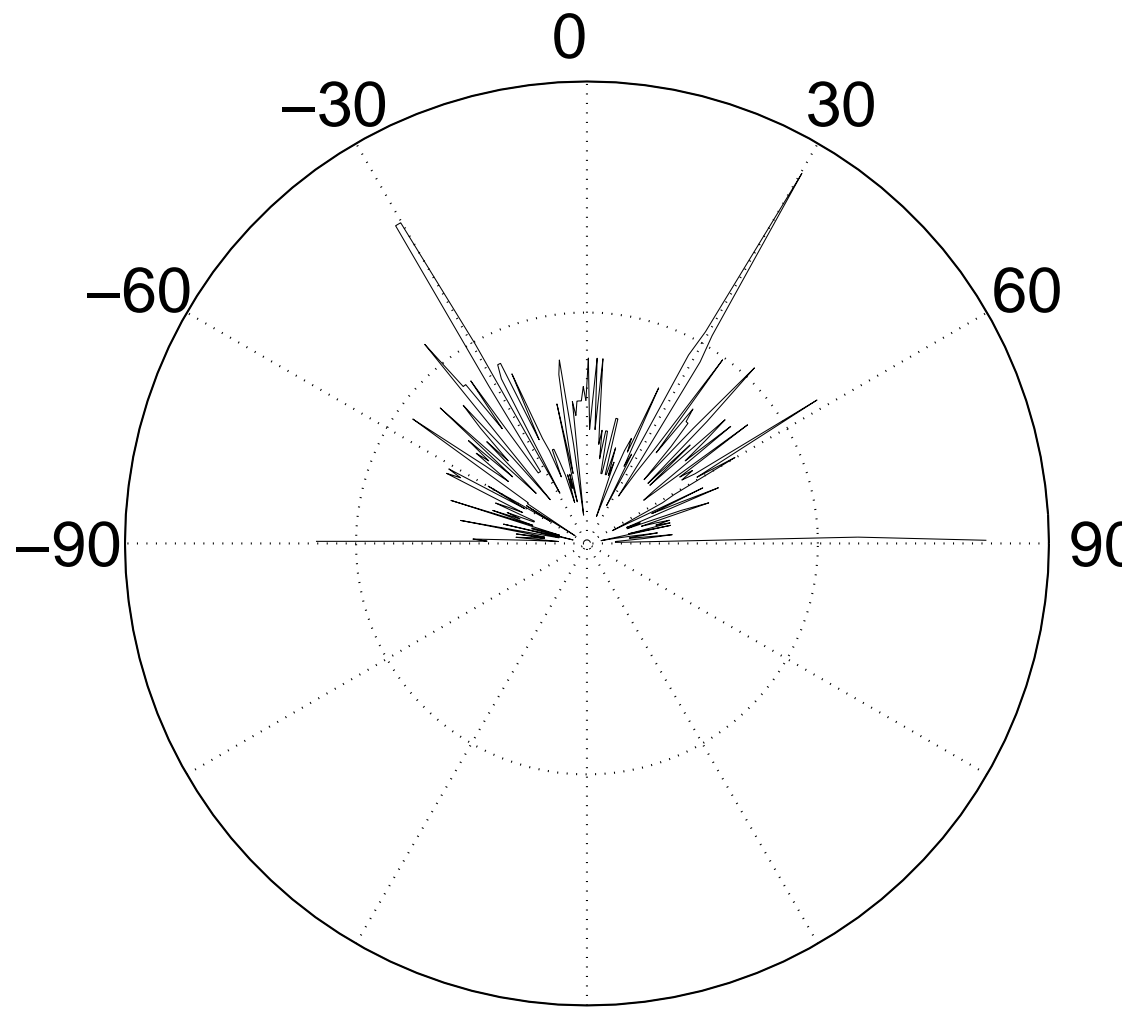

FIG. 9: Polar diagram of the distribution $p(\theta)$ of contact angles in a CD packing with $\theta_{c}=0$. The zero angle refers to the vertical direction. The angles are in degrees. 


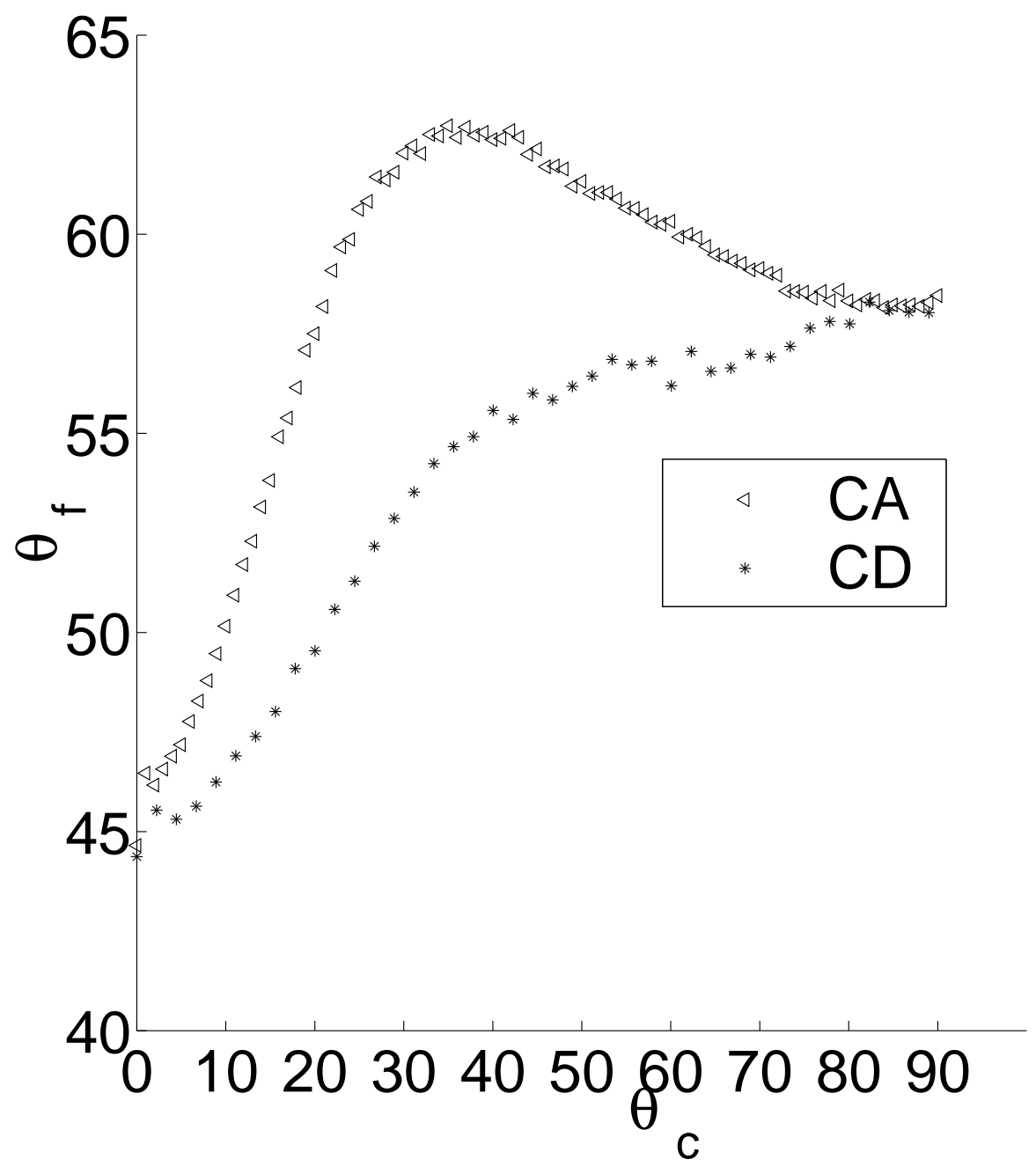

FIG. 10: The major principal directions $\theta_{f}$ of the fabric tensor as a function of critical angle. The angles are in degrees. 


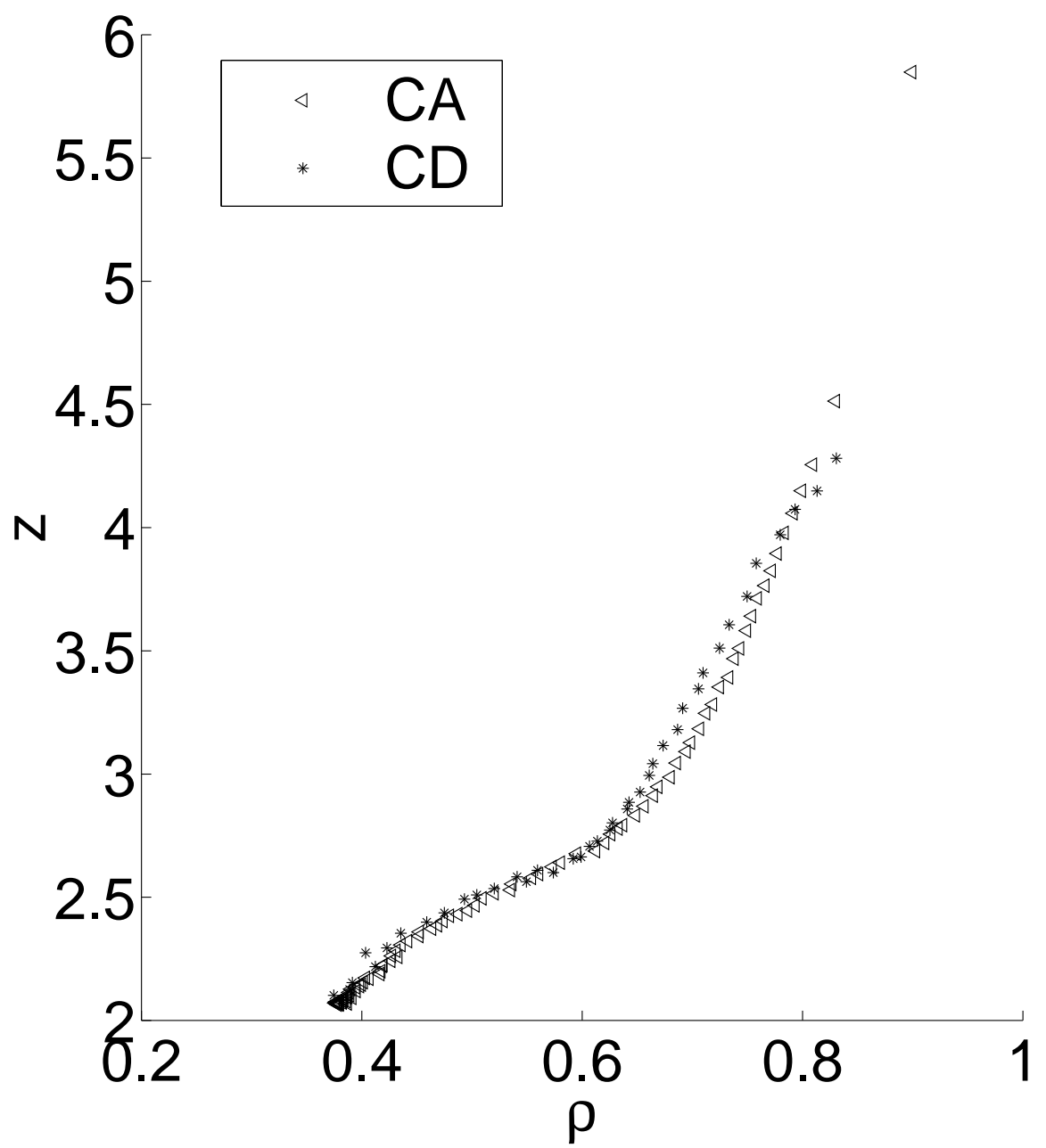

FIG. 11: Coordination numbers as a function of solid fraction $\rho$. 


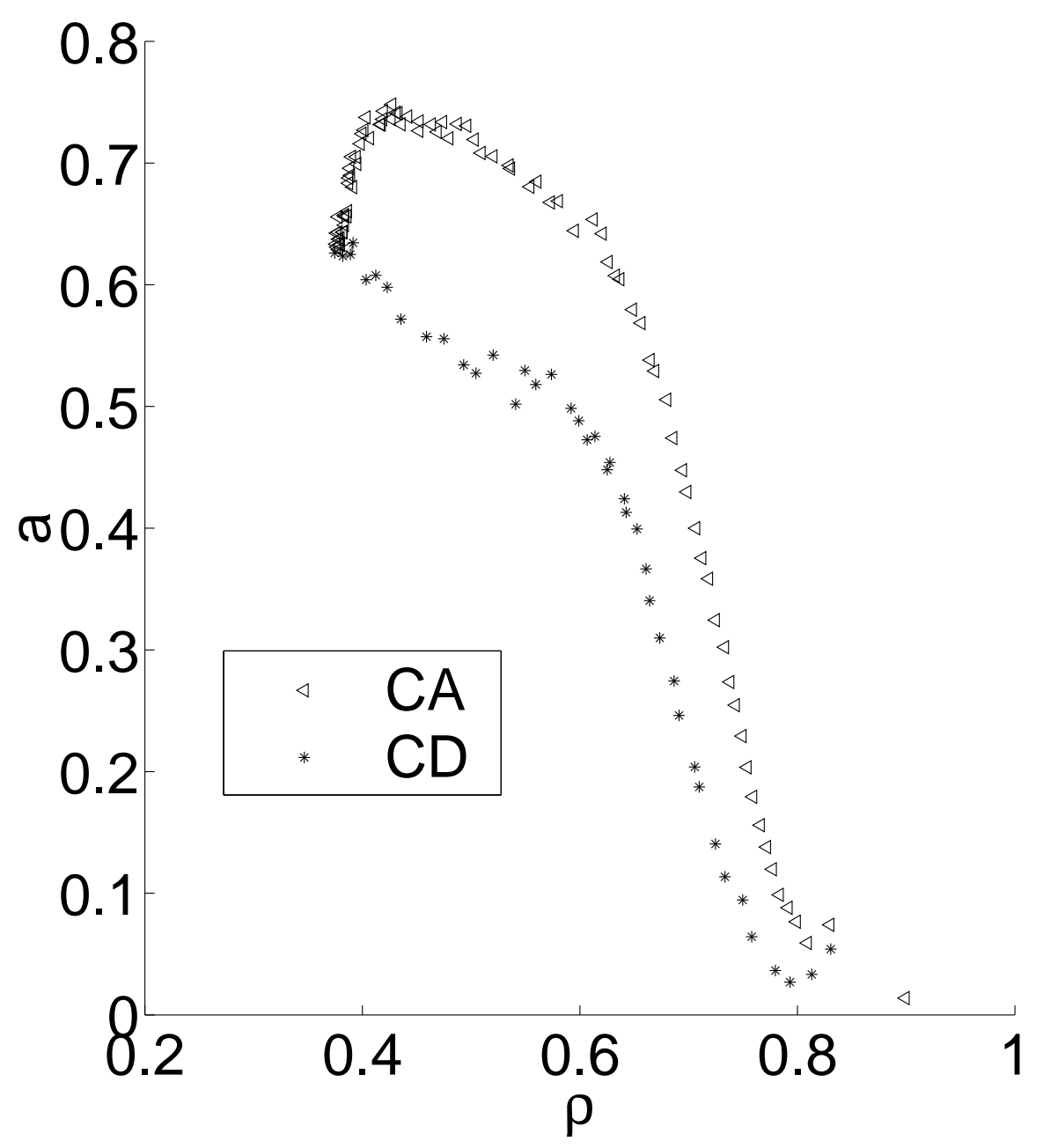

FIG. 12: Anisotropies $a$ as a function of solid fraction. 


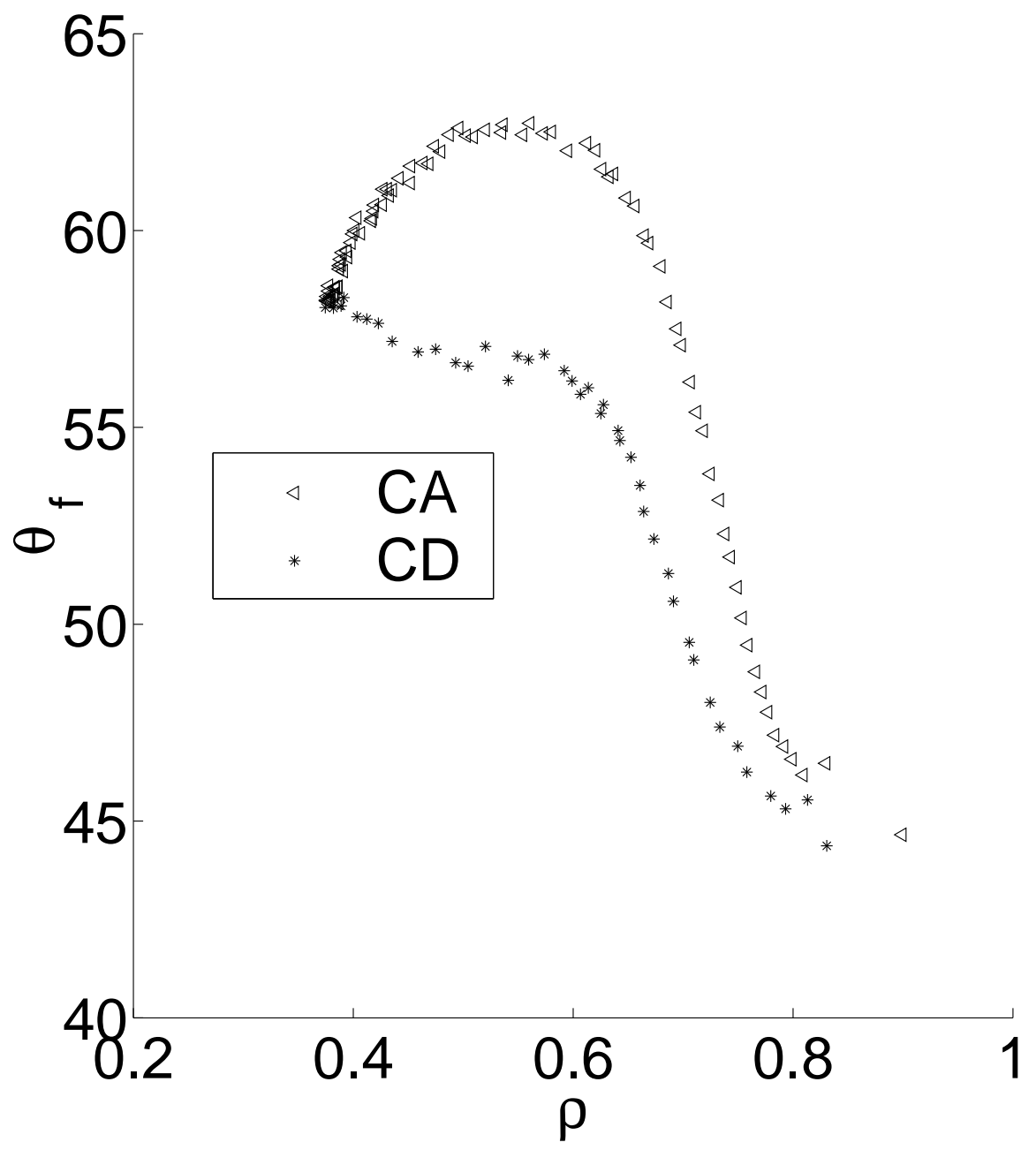

FIG. 13: The major principal directions $\theta_{f}$ (in degrees) of the fabric tensor as a function of solid fraction. 
(a)

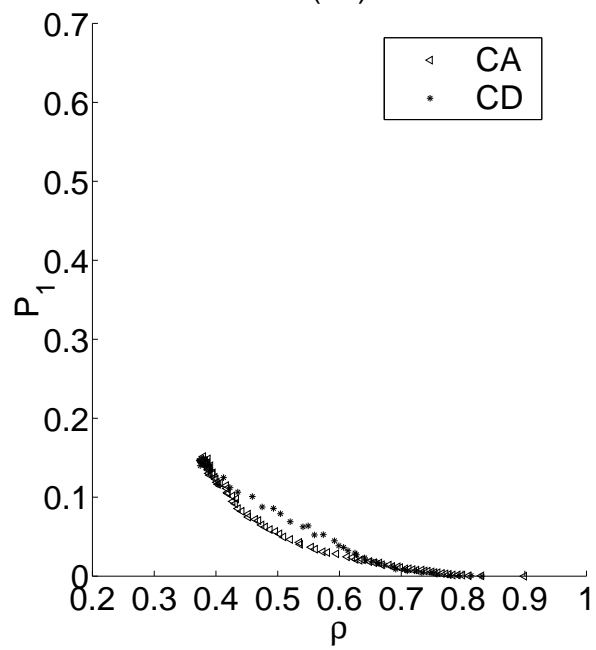

(c)

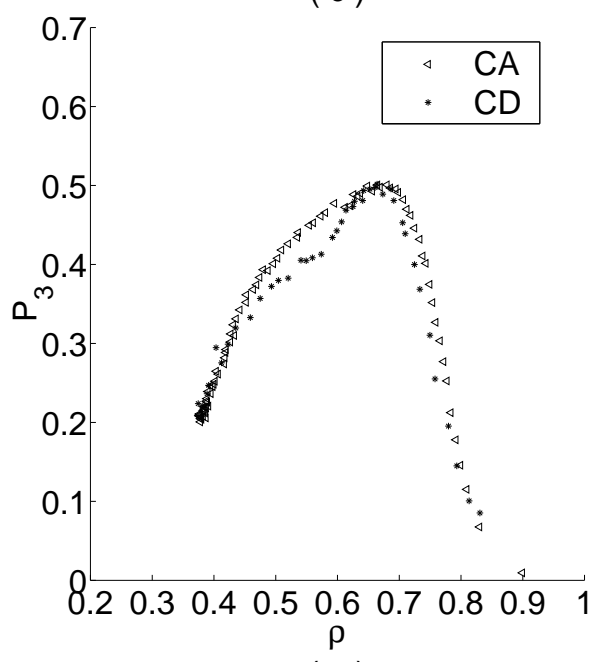

(e)

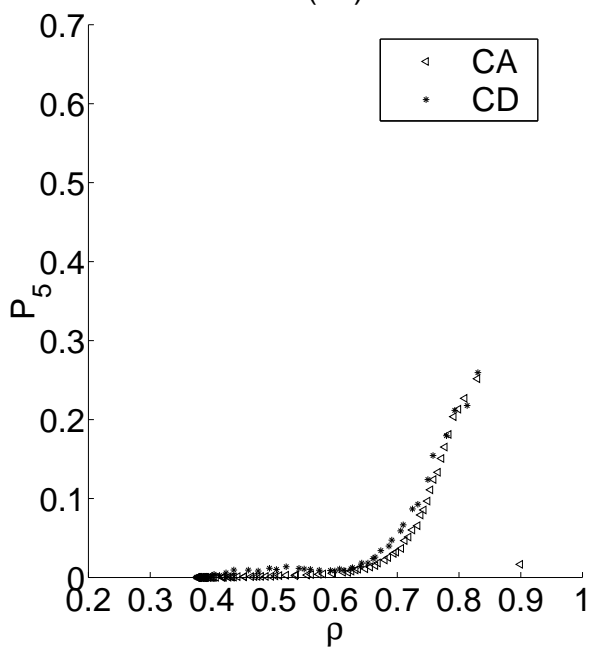

( b )

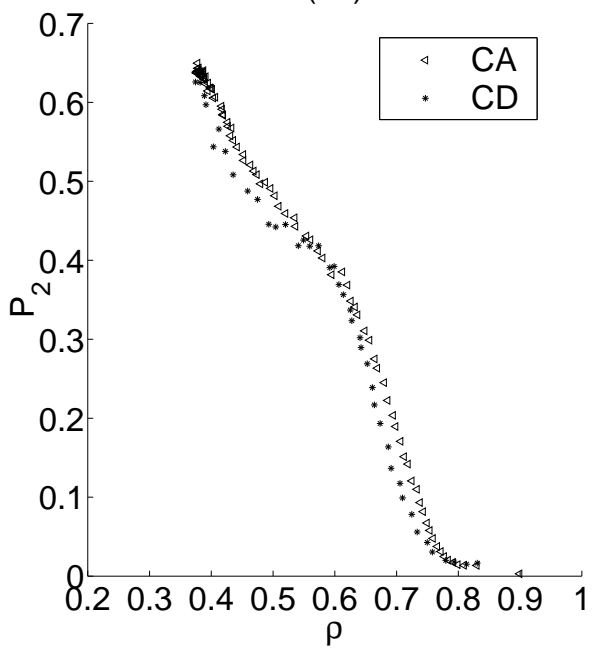

(d)

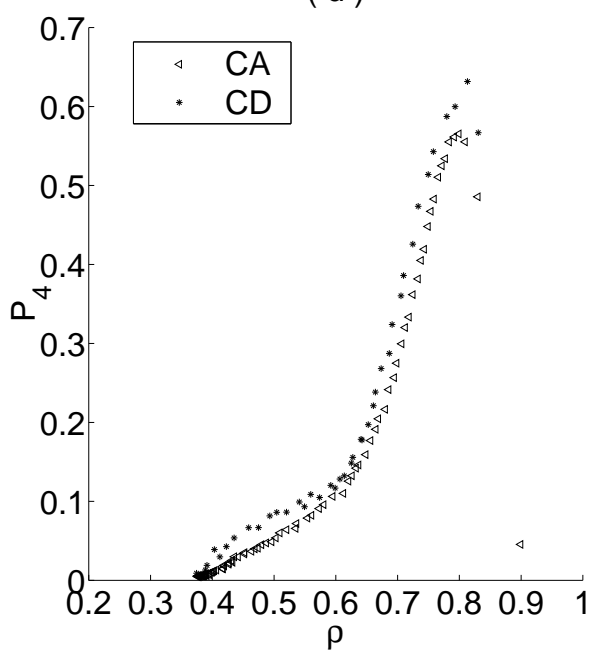

(f)

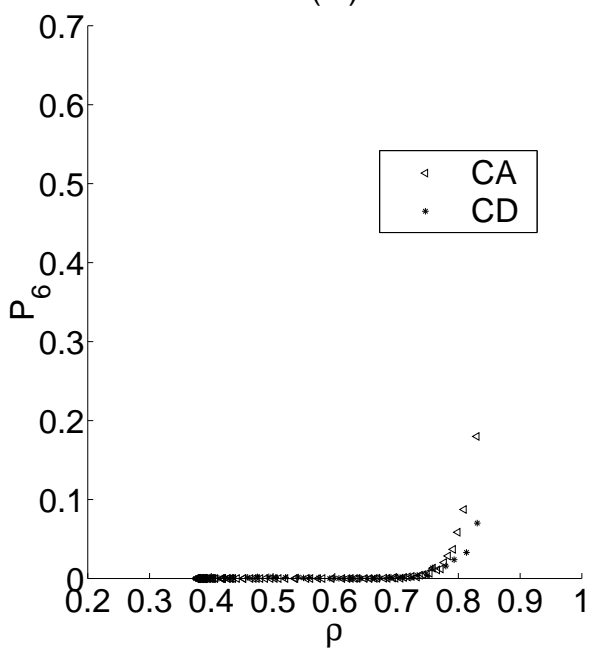

FIG. 14: The connectivity numbers $P_{1}, \cdots, P_{6}$ as a function of solid fraction. 
(a)

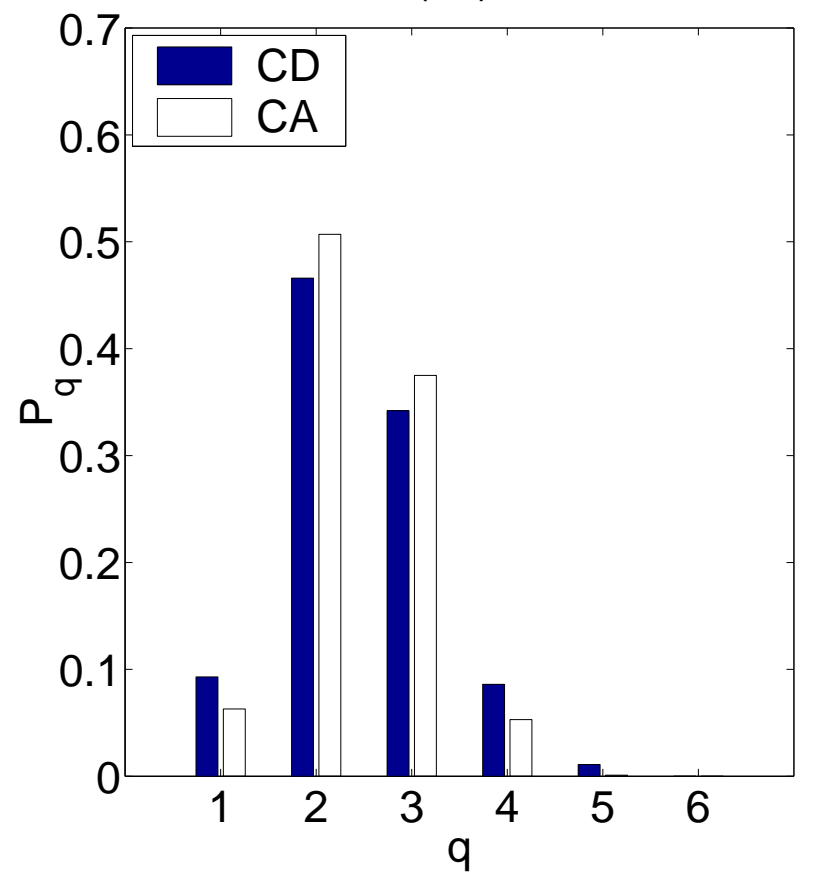

(b)

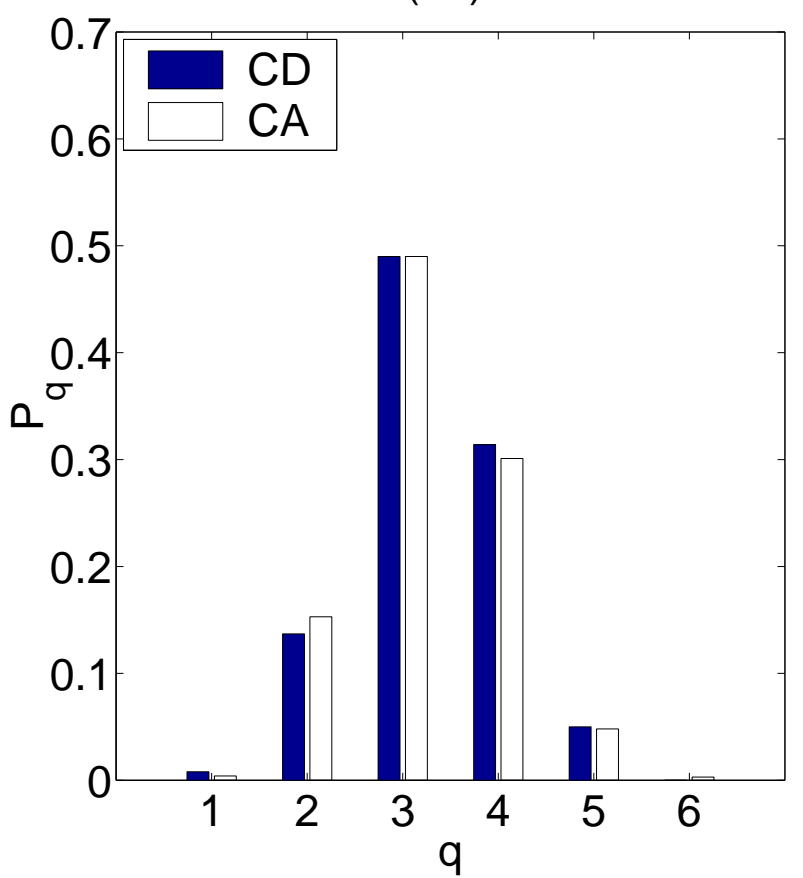

(c)

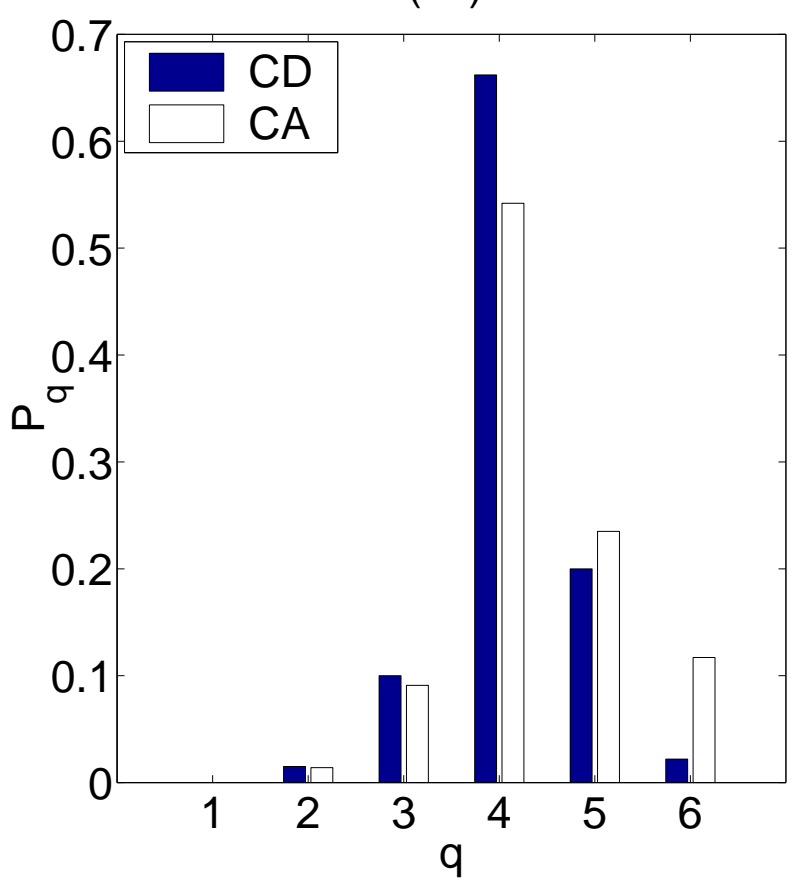

FIG. 15: The connectivity diagram of CA and CD packings packings at three different values of solid fraction: (a) $\rho=0.5$, (b) $\rho=0.7$ and (c) $\rho=0.8$. 